\title{
Short-term variations of optic coherence tomography findings in mild and severe chronic obstructive pulmonary disease
}

\author{
Nalan Ogan ${ }^{1} \cdot$ Pinar Altiaylik Ozer Mehmet Fatih Kocamaz $^{2} \cdot$ E. Eylem Akpinar ${ }^{1} \cdot$ Ayse Baha $^{3} \cdot$ \\ Esen Sayin Gulensoy ${ }^{1}$
}

Received: 26 December 2018 / Revised: 4 May 2019 / Accepted: 31 July 2019 / Published online: 4 October 2019

(c) The Author(s), under exclusive licence to The Royal College of Ophthalmologists 2019

\begin{abstract}
Objective To evaluate the short-term changes in subfoveal choroidal thickness (SFCT), ganglion cell complex (GCC) analysis, and retinal nerve fiber length (RNFL) of patients with chronic obstructive pulmonary disease (COPD) in a 3-month follow-up.

Materials and methods Forty-eight patients with COPD (96 eyes) and 40 control subjects (80 eyes) were enrolled in our study. COPD patients were grouped according to disease severity as Group 1 (mild-moderate) and Group 2 (advanced). GCC, RNFL, and SFCT analysis by Cirrus SD-OCT were obtained for all eyes, in two consecutive examinations with a 3-month interval.

Results SFCT in Group 2 was lower than Group 1 and control group in the initial and 3rd month examination $(p<0.001$, respectively). Inferior RNFL in Group 2 were lower than control group in the initial and 3rd month examination $(p=0.002$, $p<0.001$, respectively) Temporal RNFL were lower in Group 2 than Group 1 in 3rd month examination $(p=0.009)$. Average, superior, superotemporal, inferior, and inferonasal GCC analyses of the Group 2 were lower than control group both in the initial and 3rd month examination ( $p=0.001, p<0.001$, respectively) SFCT, average, and superior GCC of Group 2 were significantly reduced during the 3-month follow-up $(p<0.001)$.

Conclusion Hypoxia is thought to be the underlying mechanism in COPD, which may influence retinal and choroidal OCT parameters. Decrease in blood flow of optic nerve head, increased vascular resistance, and reduced blood flow in choroid may affect the visual ability in these patients, which should be kept in mind during their follow-up.
\end{abstract}

\section{Introduction}

Altered respiratory response to inhaled agents build up the main pathological mechanism in the development of chronic obstructive pulmonary disease (COPD) [1]. It is regarded as a systemic disorder that includes a low-grade systemic inflammation that goes with various systemic comorbidities $[2,3]$.

Nalan Ogan

nalanogan@gmail.com

1 Department of Chest Diseases, Ufuk University Faculty of Medicine, Ankara, Turkey

2 Department of Ophthalmology, Ufuk University Faculty of Medicine, Ankara, Turkey

3 Department of Chest Diseases, Kyrenia Akcicek National Hospital, Kyrenia, Cyprus
Chronic hypoxia and associated inflammatory cytokines initiate many pathways resulting in systemic effects of COPD [2, 4]. Deteriorating pulmonary functions and worsening disease status increase the risk of alveolar hypoxia and appearance of systemic co-morbidities [5].

The retinal and choroidal vascular structures highly reflect the changes in systemic vascular disorders of the body. The effects of obstructive sleep apnea syndrome on retina and choroidal microvasculature are already defined in the literature, which manifests systemic pathophysiological changes similar to those of COPD, including chronic hypoxemia, systemic inflammation, vascular dysregulation, and increased sympathetic activity [6, 7].

Chronic hypoxemia and associated inflammation are found to effect retinal and choroidal blood flow [8]. Optical coherence tomography (OCT) is a noninvasive and quantitative technique that is widely used for the diagnosis and follow-up of ocular involvement in systemic diseases, since it objectively evaluates retina and choroid [9]. SD-OCT is 
now acquiring a great role with its greater tissue penetrance, for the assessment of retinal and choroidal structural alterations in systemic diseases [10]. With the help of its improved image resolution, spectral-domain OCT measures the ganglion cell-inner plexiform layer (GCIPL) in the macular area which is now regarded as an important marker in OCT for detecting and monitoring the neuronal degeneration [11].

The new diagnostic role of OCT in COPD has been reported in previous studies [8, 12, 13]. Peripapillary choroid in the inferior segments of patients with COPD was found to be significantly thinner than other peripapillary choroid segments and also thinner than those of control patients [8]. The authors reported no differences in average peripapillary and subfoveal choroidal thickness (SFCT) values of patients with COPD compared with controls [8].

In the only study which correlated the disease severity and OCT findings of COPD patients, Gok et al. found no significant difference in macular choroidal thickness of patients with mild to moderate and severe COPD compared with matched controls [13]. Similar results were obtained by Ugurlu et al, who also reported no significant difference in subfoveal choroid thickness between a group of male COPD patients and male controls [12]. In all of these studies, the choroidal thinning in COPD patients was attributed to hypoxia and vascular endothelial dysfunction.

The purpose of our study is to determine the effect of COPD severity on retinal and choroidal structure, by monitoring the short-term changes in OCT parameters detected in the initial and 3rd month examination. To the best of our knowledge, this is the first study evaluating the short-term changes of OCT parameters in COPD patients.

\section{Patients and methods}

This was a retrospective study that was conducted under a protocol approved by the Ufuk University Faculty of Medicine Ethics Committee and was in accordance with the ethical standards stated in the 1964 Declaration of Helsinki. Informed consent was obtained from all participants.

Forty-eighty patients with COPD with at least 5 years of disease duration without any history of eye disorders other than mild cataracts were enrolled in the study (COPD group). COPD diagnosis was based on the spirometry results-forced expiratory volume in $1 \mathrm{~s} /$ forced expiratory vital capacity (FEV1/ FVC) $<70 \%$ and reversibility test negative patients were diagnosed as COPD.

The FEV1, forced vital capacity (FVC) and FEV1/FVC were measured in all patients in stable period and controls using a ZAN 300 platform (nSpire Health GmbH, Oberthulba, Germany)
Patients with COPD were classified into following four groups according to the Global Initiative for Chronic Obstructive Lung Disease (GOLD) classification, exercise capacity (modified Medical Research Council (mMRC) and the British Medical Research Council values) and risk score (exacerbation frequency $=\mathrm{EF}$ ): stage A (mild airflow limitation, FEV1/ FVC $<70 \%$, FEV $1 \leq 80 \%, \mathrm{EF} \leq 2, \mathrm{mMRC} \leq$ 2); stage B (worsening limitation, FEV1/FVC $<70 \%, 50 \% \leq$ FEV $1<80 \%, \mathrm{EF} \leq 2, \mathrm{mMRC} \geq 2$ ); stage $\mathrm{C}$ (severe limitation, $\mathrm{FEV} 1 / \mathrm{FVC}<70 \%, 30 \% \leq \mathrm{FEV} 1<50 \%, \mathrm{EF} \geq 2, \mathrm{mMRC} \leq$ 2 ); or stage D (very severe limitation, FEV1/FVC $<70 \%$, FEV $1<30 \%, E F \geq 2, m M R C \geq 2$ ) [14]. Patients in stage A-B constituted Group 1 (mild-moderate COPD) and those in stage $\mathrm{C}-\mathrm{D}$ were determined to be in Group 2 (severe COPD).

Exclusion criteria for the study were: best-corrected visual acuity $(\mathrm{BCVA})<8 / 10$, history of previous intraocular/refractive surgery/retinal laser photocoagulation, glaucoma, amblyopia, optic neuropathy, significant cataracts, and refractive errors (RE) of spherical equivalent more than \pm 3.0 diopters, axial length (AL) over $25 \mathrm{~mm}$, choroidal neovascularization, or myopic maculopathy, any opacities of the optic media and low image quality due to errors of fixation. Chronic systemic diseases like diabetes, hypertension, or cardiovascular diseases, patients under 40 years of age and cases during the acute exacerbations of COPD were not included in the study. The control group $(n=40)$ consisted of age and sex-matched healthy patients, without any diagnosis of previous or current COPD and no history of smoke or chronic disease (Diabetes mellitus, hypertension or cardiovascular diseases) in order to avoid possible effects on OCT parameters.

Medical records of the study and control groups that included ophthalmic examinations were retrospectively evaluated. All participants underwent a complete ophthalmological evaluation, including BCVA, slit lamp biomicroscopy, intraocular pressure (IOP) measurement, fundus examination, RE examination, and AL measurements. Blood gas analysis had been performed for patients with COPD and oxygen saturation was measured by pulse oximetry.

All the OCT measurements were performed twice for each COPD patient in the study. First measurement was done during their first clinical examination and the second measurement was done in the 3rd month control visit. Retinal nerve fiber length (RNFL) measurements, ganglion cell complex (GCC) analysis and SFCT measurements were obtained for control group patients in two consecutive visits with a 3-month interval.

OCT scan was performed after pupil dilation with tropicamide $1 \%$ using a Cirrus HD 500 spectral OCT platform (Carl Zeiss Meditec, Dublin, CA, USA). Only high-quality images (signal strength $\geq 7$ ) were selected for the study. The 
peripapillary RNFL thickness was measured by an optic disc cube scan protocol $(200 \times 200$ pixels $)$ in a $6 \times 6 \mathrm{~mm}^{2}$ area centered on the optic disc. The macular cube scan $512 \times 126$ protocol was used to evaluate $6 \times 6-\mathrm{mm}^{2}$ area centered on the fovea in terms of GCC analysis. The algorithm of the GCC analysis protocol based on the identification of the macular Ganglion cell-Inner Plexiform Layer (GCIPL), from the outer boundary of the RNFL to the outer limit of the inner plexiform layer.

The average RNFL thickness (and those of the four quadrants; superior, nasal, inferior, and temporal), the thicknesses of the six wedge-shaped sectors of the GCC were automatically calculated and reported. Scans with misalignment, segmentation failure, or decentration of the measurement circle, artefacts induced by eye movement during scan and dropout or missing parts on deviation maps were excluded from the analysis.

All SFCT measurements were performed independently by two different ophthalmologists (PAO and MFK) who evaluated the subfoveal sections. The ophthalmologists performing the measurements were blind to the group of the COPD subject.

The distance between the outer hyperreflective border of retina pigment epithelium and inner scleral surface was measured to optimize the accuracy of SFCT measurements (Fig. 1). The averaged data detected by two clinicians were used in statistical analysis. All interobserver differences were calculated to be within $10 \%$ of the mean values.

\section{Statistical analysis}

Statistical analyses were performed with IBM SPSS for Windows Version 22.0 software. Numerical variables were expressed as mean \pm standard deviation or median [min-max] as appropriate. Categorical variables were summarized as numbers and percentages. Normality of the continuous variables was tested by Shapiro-Wilk's test. Levene test was used to show the homogeneity of variances. Independent samples $t$ test or Mann-Whitney $U$ test was used to determine the difference between two independent groups. More than two independent groups were compared by one way ANOVA or Welch ANOVA as appropriate. Post hoc comparisons were done by Tukey HSD or Games Howell test. Kruskal-Wallis test was used to show differences between groups when parametric test assumptions were not met. Categorical variables were compared by $\chi^{2}$ test or Fisher exact test. Repeated measures ANOVA was performed to show between and within group differences according to ocular parameters. Pairwise comparisons were done by Bonferroni test. The degrees of relation between variables were measured with pearson correlation or spearman correlation analysis. A $p$-value $<0.05$ was considered statistically significant.

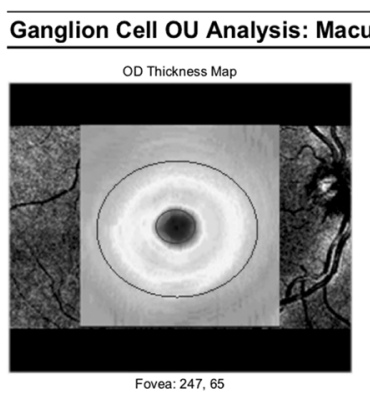

Fovea: 247,65
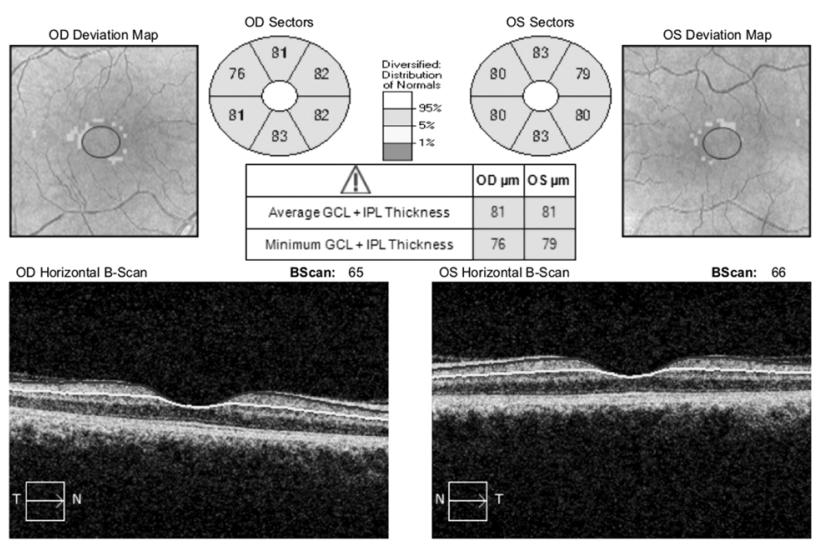
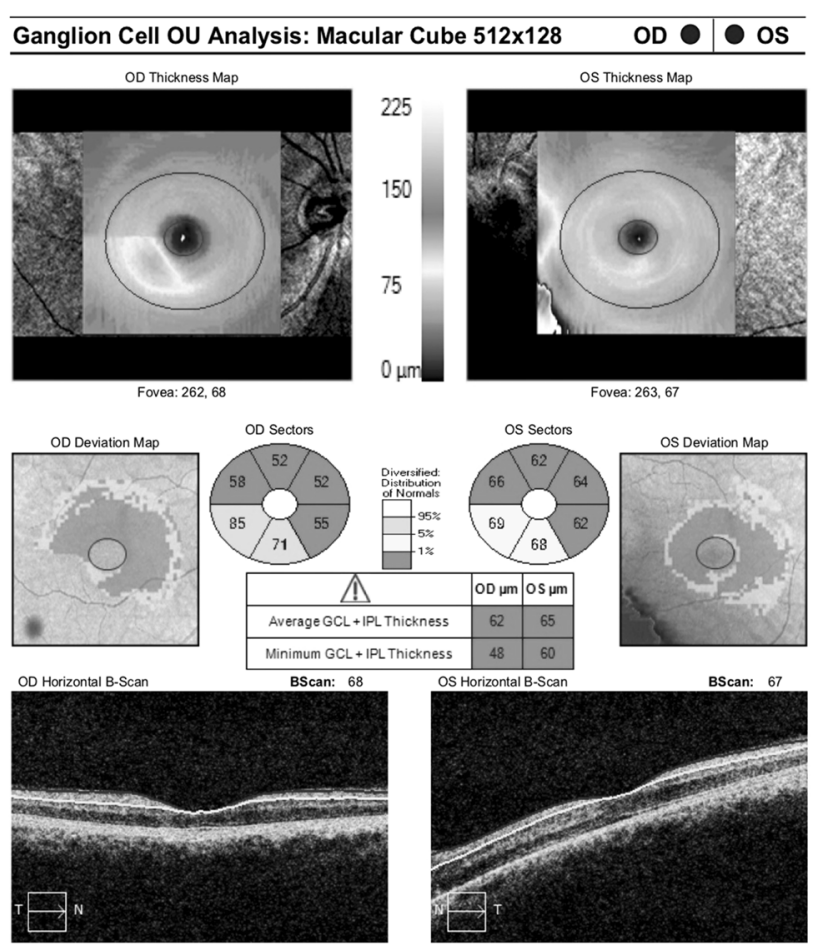

Fig. 1 Comparison of ganglion cell analysis (GCC) results of a normal and a Group 2 (Gold C-D) patient 


\section{Results}

This study included 48 COPD patients (38 males 79.1\%), 10 females (20.8\%); mean age: $65.8 \pm 11.8$ years) and 40 age and sex-matched controls 17 males (42.5\%), 23 females (57.5\%); mean age: $67 \pm 6.7$ years). Table 1 shows demographics of the patients and control group enrolled in the study. There were no statistically significant differences between the COPD and control groups in terms of age, BCVA, or IOP.

Arterial gas saturation and pulmonary function test results of Group 1 and Group 2 patients are shown in Table 2. Although there was a difference in oxygen saturation between the two groups, it was not statistically significant.

OCT parameters of Group 1 and 2 including RNFL thickness, GCC measurements and SFCT values for COPD patients were compared with the control group. Comparison of these measurements in the initial and 3rd month control visits were the other compared data among Group 1 and 2 .

Table 3 shows the RNFL measurements of Groups 1, 2, and control group. Inferior segment RNFL thickness values of the Group 2 COPD were lower than control group both

Table 1 Demographic characteristics of COPD and control subjects

\begin{tabular}{llllr}
\hline & $\begin{array}{l}\text { Control } \\
(n=40)\end{array}$ & $\begin{array}{l}\text { GOLD A-B } \\
(n=14)\end{array}$ & $\begin{array}{l}\text { GOLD C-D } \\
(n=34)\end{array}$ & $p$ \\
\hline Age (year) & $67.0 \pm 6.7$ & $62.1 \pm 12.5$ & $69.5 \pm 11.2$ & 0.176 \\
Sex (M/F) & $17 / 23$ & $10 / 4$ & $28 / 6$ & 0.001 \\
Smoking status & $(42.5 \% / 57.5 \%)$ & $(71.4 \% / 28.6 \%)$ & $(82.4 \% / 17.6 \%)$ & \\
$\quad$ & & & \\
Non-smoker & - & - & $3(8.8 \%)$ & 0.335 \\
Active & - & $4(26.8 \%)$ & $8(23.5 \%)$ & \\
Ex-smoker & - & $10(71.4 \%)$ & $23(67.6 \%)$ & \\
Cigarette packet/year & - & $40(5-120)$ & $40(4-100)$ & 0.266 \\
BMI & - & $27.0 \pm 5.8$ & $26.1 \pm 6.0$ & 0.643 \\
COPD year & - & $5(1-15)$ & $6[2-20]$ & 0.039 \\
Exacerbation per year & - & $0(0-2)$ & $2[1-6]$ & $<\mathbf{0 . 0 0 1}$ \\
mMRC & - & $1(1-2)$ & $2[1-4]$ & 0.002 \\
LTOT & - & $4(28.6 \%)$ & $17(50 \%)$ & 0.298 \\
\hline
\end{tabular}

$B M I$ body mass index, $m M R C$ Modified Medical Research Council, LTOT long-term oxygen treatment

Bold values indicate statistical significance $p<0.05$

Table 2 Arterial gas saturation and pulmonary function test of COPD groups

\begin{tabular}{llll}
\hline & GOLD A-B $(n=14)$ & GOLD C-D $(n=34)$ & $p$ \\
\hline SaO2, \% & $95.5(4.7-98)$ & $88.8(2.6-98)$ & 0.064 \\
FVC (\%) & $70.6 \pm 19.9$ & $60.7 \pm 15.9$ & 0.092 \\
FVC (liter) & $2.5 \pm 0.8$ & $1.9 \pm 0.7$ & 0.015 \\
FEV1 (\%) & $55.5 \pm 20$ & $45.4 \pm 18.8$ & 0.124 \\
FEV1 (liter) & $1.6 \pm 0.6$ & $1.1 \pm 0.5$ & 0.005 \\
FEV1/FVC & $62.6 \pm 13.0$ & $56.4 \pm 16.9$ & 0.257 \\
\hline
\end{tabular}

$\mathrm{SaO} 2$ arterial oxygen saturation, $F V C$ forced vital capacity, FEV1 forced expiratory volume in $1 \mathrm{~s}$ during the initial examination $(p=0.002)$ and in the $3 \mathrm{rd}$ month examination $(p<0.001)$ (Fig. 1). Temporal RNFL thickness values were lower in Group 2 patients compared with Group 1 in the 3rd month examination $(p=0.009)$.

Average GCC analysis of the Group 2 COPD were lower than control group during the initial examination $(p=$ $0.001)$ and in the 3rd month examination $(p<0.001)$ (Fig. 2). Table 4 shows the GCC and SFCT measurements of Groups 1,2, and control group. The average subfoveolar choroidal thickness (SFCT) measurements of the Group 2 was lower than both Group 1 and Control Group during the initial examination $(p<0.001)$ and in the $3 \mathrm{rd}$ month examination $(p<0.001)$ (Figs. $3-5)$.

Table 5A, B gives the results of correlation analysis investigating the effects of potential factors on OCT findings. In the initial examination of Group 1 patients, mMRC value is negatively correlated with nasal and temporal RNFL measurements in addition to inferotemporal GCC and SFCT results. In the 3rd month examination of Group 1 patients, mMRC value is negatively correlated with inferotemporal RNFL measurements, whereas FVC and FEV1 values have a positive correlation with ARNFL.

In the initial examination of Group 2 patients, number of smoked cigarettes (packets) per year and attacks per year are found to have a negative correlation with ARNFL values. Attacks per year are also found to have a negative

Table 3 RNFL measurements of COPD and control subjects

\begin{tabular}{lllll}
\hline & $\begin{array}{l}\text { Control } \\
(n=40)\end{array}$ & $\begin{array}{l}\text { GOLD A-B } \\
(n=14)\end{array}$ & $\begin{array}{l}\text { GOLD C-D } \\
(n=34)\end{array}$ & $p$ \\
\hline ARFNL & & & & \\
$\quad$ Initial & $94.7 \pm 14.8$ & $99.1 \pm 5.3$ & $93 \pm 7.5$ & 0.233 \\
3rd month & $94.7 \pm 14.8$ & $97.6 \pm 5.3$ & $92.8 \pm 6.5$ & 0.382 \\
$p$ & 1.000 & 0.366 & 0.868 & \\
IRFNL & & & & $0.002^{\mathrm{a}}$ \\
Initial & $108.9 \pm 4.6$ & $107.7 \pm 4.8$ & $100 \pm 16.7$ & $<0.001^{\mathrm{a}}$ \\
3rd month & $108.9 \pm 4.6$ & $106.1 \pm 4.8$ & $103.1 \pm 5.4$ & \\
$p$ & 1.000 & 0.601 & 0.105 & 0.268 \\
SRFNL & & & & 0.350 \\
Initial & $92.5 \pm 4.9$ & $93 \pm 5.3$ & $90.7 \pm 5.8$ & \\
3rd month & $92.5 \pm 4.9$ & $93.2 \pm 4.8$ & $91 \pm 6.1$ & 0.400 \\
$p$ & 1.000 & 0.895 & 0.778 & 0.050 \\
NRNFL & & & $84 \pm 6.4$ & \\
Initial & $85.3 \pm 5.7$ & $86.4 \pm 5.3$ & $82.8 \pm 6.2$ & 0.058 \\
3rd month & $85 \pm 5.8$ & $87.3 \pm 5.4$ & 0.283 & \\
$p$ & 0.790 & 0.624 & $75.8 \pm 5.6$ & \\
TRNFL & & & $75.1 \pm 5.5$ & 0.431 \\
Initial & $77.1 \pm 4.7$ & $79.6 \pm 4.1$ & & \\
3rd month & $77.1 \pm 4.6$ & $79.8 \pm 2.8$ & 0.922 & \\
$p$ & 1.000 & & & \\
\hline
\end{tabular}

${ }^{\mathrm{a}}$ Gold C-D different from control group

${ }^{\mathrm{b}}$ Gold C-D different from Gold A-B

$A R N F L$ average retinal nerve fiber length, IRNFL inferior retinal nerve fiber length, $S R N F L$ superior retinal nerve fiber length, $N R N F L$ nasal retinal nerve fiber length, $T R N F L$ temporal retinal nerve fiber length 

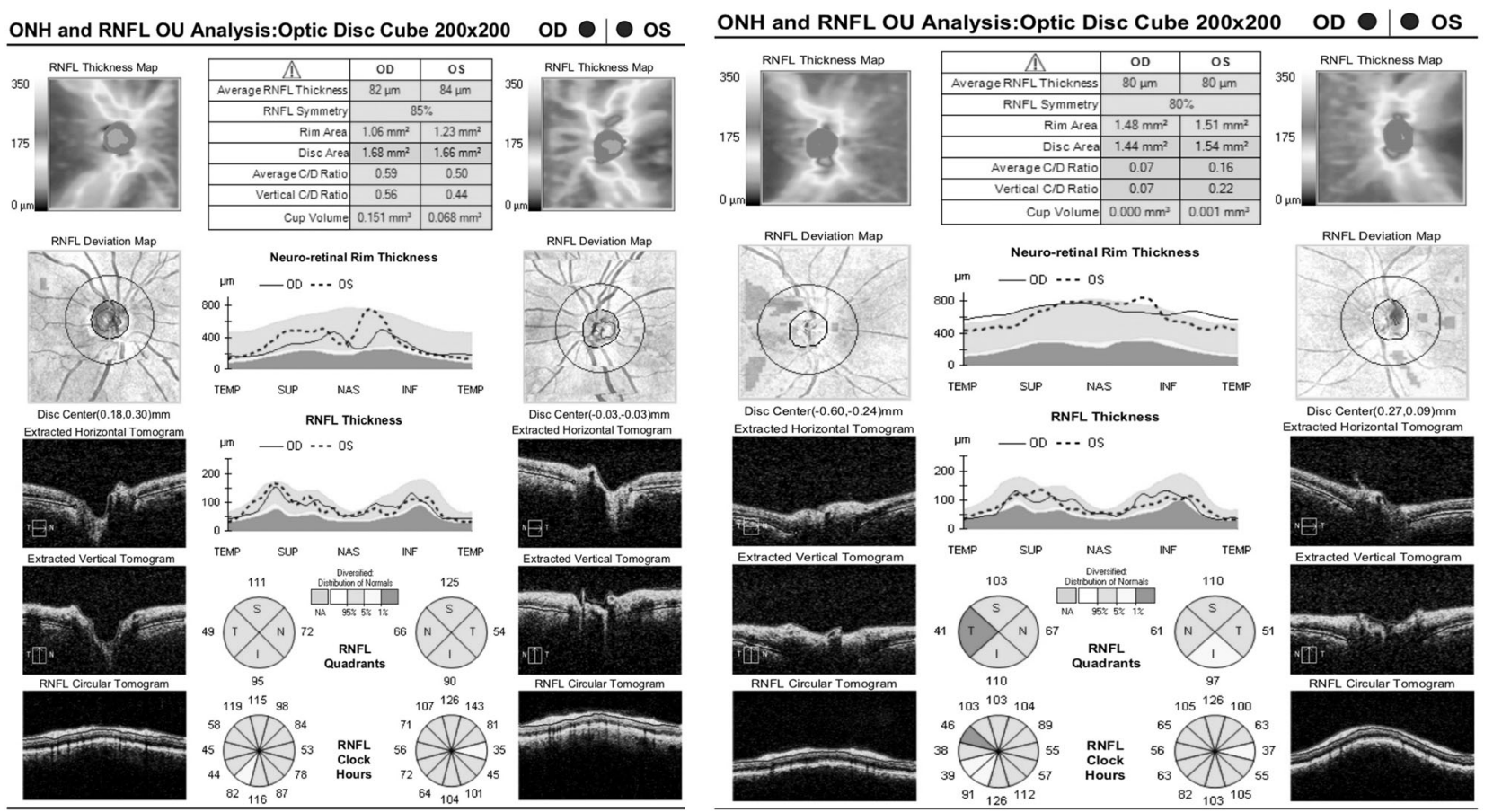

Fig. 2 Comparison of retinal nerve fiber length (RNFL) analysis results of a normal and a Group 2 (Gold C-D) patient

correlation with NRNFL and TRNFL values in addition to A-GCC, S-GCC, SN-GCC, ST-GCC, I-GCC, IN-GCC, ITGCC, and SFCT results. mMRC has a negative correlation with IRNFL, SRNFL, NRNFL, ST-GCC, and SFCT.

In the 3rd month evaluation of Group 2 patients, it was seen that COPD duration has a negative correlation with all GCC measurements except IT-GCC. Number of attacks per year is observed to have a significant negative correlation with all the GCC values and SFCT measurements, too. mMRC, FVC ( $\%$ and LT), FEV1/FVC values are found to have a positive correlation with SFCT results (Table 5A, B).

\section{Discussion}

Choroid is known to be the most vascularized section of the posterior segment of human eye and is affected in many ocular and systemic disorders [15-17]. Evaluation of the choroidal thickness is possible now with common use of SD-OCT in routine ophthalmology practice. Many systemic disorders like systemic sclerosis, acromegaly, hypercholesterolemia, and diabetes are reported to influence choroidal thickness [18-21]. Increasing age is known to be with reduced choroidal thickness measurements [17].

Choroidal thickness is regarded to be affected by choroidal blood circulation that is highly dependent on systemic blood pressure, intraoculary pressure, mean oculary perfusion pressure, nitric oxide, catecholamines, and vascular autoregulation [22]. SFCT alterations in systemic diseases had been the subject of many studies to provide important information reflecting systemic cardiovascular health [19, 23-25].

Hypoxia has a direct role on induction of endothelial dysfunction, and activation of choroidal vascular autoregulatory mechanisms has a preventive role in exaggerated alterations of choroidal thickness [26]. In our study, SFCT values were found to be significantly lower in patients with severe COPD compared with mild cases and control group patients. Similarly, Ozcimen et al. [8] and Ugurlu et al. [12] also studied choroidal thickness in subjects with COPD, and SFCT values were found to be thinner than in the control groups in both of the studies-but none were reported to have a statistical significance [8, 12].

SFCT values were determined to show a statistically significant reduction from the initial values in severe COPD group, when measured after 3 months from the initial visit. Hypoxia induced chronic vascular resistance in COPD patients and a resultant progressive impairment in ocular blood flow may be the leading cause of this serial choroidal thinning. Number of attacks per year is shown to have a significant negative correlation with SFCT measurements in 3rd month follow-up examination of severe COPD patients, which is probably the cause of reduced ventilatory capacity in these cases. The positive correlation of mMRC, FVC (\% and LT) and FEV1/FVC values with SFCT results is another important finding in this group, supporting this hypothesis.

Evaluation of peripapillary nerve fiber layer thickness with SD-OCT is a good monitoring tool for axonal integrity 
Table 4 GCC and SFCT measurements of COPD and control subjects

\begin{tabular}{|c|c|c|c|c|}
\hline & $\begin{array}{l}\text { Control } \\
(n=40)\end{array}$ & $\begin{array}{l}\text { GOLD A-B } \\
(n=14)\end{array}$ & $\begin{array}{l}\text { GOLD C-D } \\
(n=34)\end{array}$ & $p$ \\
\hline \multicolumn{5}{|l|}{ A-GCC } \\
\hline Initial & $86.8 \pm 4.3$ & $85.5 \pm 5.1$ & $80.8 \pm 9.2$ & $0.001^{\mathrm{a}}$ \\
\hline 3rd month & $86.8 \pm 4.3$ & $85.1 \pm 5.7$ & $79.4 \pm 10.5$ & $<0.001^{\mathrm{a}}$ \\
\hline$p$ & 1.000 & 0.399 & $<0.001$ & \\
\hline \multicolumn{5}{|l|}{ S-GCC } \\
\hline Initial & $88.3 \pm 4.5$ & $86.6 \pm 5.2$ & $82.5 \pm 9$ & $0.001^{\mathrm{a}}$ \\
\hline 3rd month & $88.3 \pm 4.5$ & $86.6 \pm 5.2$ & $81.9 \pm 9.8$ & $0.001^{\mathrm{a}}$ \\
\hline$p$ & 1.000 & 1.000 & $<0.001$ & \\
\hline \multicolumn{5}{|l|}{ ST-GCC } \\
\hline Initial & $89.9 \pm 4.5$ & $88.2 \pm 5$ & $83.7 \pm 9.4$ & $0.001^{\mathrm{a}}$ \\
\hline 3rd month & $89.9 \pm 4.4$ & $88.2 \pm 5$ & $83.7 \pm 9.4$ & $0.001^{\mathrm{a}}$ \\
\hline$p$ & 0.400 & 1.000 & 1.000 & \\
\hline \multicolumn{5}{|l|}{ SN-GCC } \\
\hline Initial & $91.1 \pm 3.4$ & $90.1 \pm 4$ & $84.5 \pm 9.6$ & $<0.001^{\mathrm{b}}$ \\
\hline 3rd month & $91.1 \pm 3.3$ & $90.1 \pm 4$ & $84.5 \pm 9.6$ & $<0.001^{\mathrm{b}}$ \\
\hline$p$ & 0.400 & 1.000 & 1.000 & \\
\hline \multicolumn{5}{|l|}{ I-GCC } \\
\hline Initial & $91.2 \pm 3.6$ & $89.2 \pm 3.3$ & $84.2 \pm 10.3$ & $<0.001^{\mathrm{a}}$ \\
\hline 3rd month & $91.2 \pm 3.6$ & $89.2 \pm 3.3$ & $84.1 \pm 10.5$ & $<0.001^{\mathrm{a}}$ \\
\hline$p$ & 1.000 & 1.000 & 0.112 & \\
\hline \multicolumn{5}{|l|}{ IN-GCC } \\
\hline Initial & $91.4 \pm 4.1$ & $89.9 \pm 4.3$ & $84.9 \pm 10.1$ & $0.001^{\mathrm{a}}$ \\
\hline 3rd month & $91.3 \pm 4.1$ & $89.9 \pm 4.3$ & $84.7 \pm 10.4$ & $0.001^{\mathrm{a}}$ \\
\hline$p$ & 0.370 & 1.000 & 0.225 & \\
\hline \multicolumn{5}{|l|}{ IT-GCC } \\
\hline Initial & $91.6 \pm 3.4$ & $90.9 \pm 4$ & $85.1 \pm 9.7$ & $<0.001^{\mathrm{b}}$ \\
\hline 3rd month & $91.6 \pm 3.3$ & $90.9 \pm 4$ & $85.1 \pm 9.7$ & $<0.001^{\mathrm{b}}$ \\
\hline$p$ & 0.144 & 1.000 & 1.000 & \\
\hline \multicolumn{5}{|l|}{ SFCT } \\
\hline Initial & $302.4 \pm 20.4$ & $311.6 \pm 17.9$ & $282.5 \pm 32.4$ & $<0.001^{\mathrm{b}}$ \\
\hline 3rd month & $302.1 \pm 20.3$ & $310.3 \pm 17.9$ & $266.3 \pm 47.3$ & $<0.001^{\mathrm{b}}$ \\
\hline$p$ & 0.922 & 0.786 & $<0.001$ & \\
\hline
\end{tabular}

${ }^{a}$ Gold C-D different from control group

${ }^{\mathrm{b}}$ Gold C-D different from all other groups

$A-G C C$ average ganglion cell complex, $S$-GCC superior ganglion cell complex, ST-GCC superotemporal ganglion cell complex, $S N-G C C$ superonasal ganglion cell complex, $I-G C C$ inferior ganglion cell complex, IN-GCC inferonasal ganglion cell complex, IT-GCC inferiotemporal ganglion cell complex SFCT subfoveal choroidal thickness

Bold values indicate statistical significance $p<0.05$

in neuro-ophthalmological disorders. Nasal and temporal RNFL quadrants are measured to be thicker compared with the inferior and superior quadrants in healthy individuals $[27,28]$. RFNL values are reduced with increasing age and also manifests a great decrease in optic neuropathies including multiple sclerosis, obstructive sleep apnea, systemic arterial hypertension, Alzheimer's, and Parkinson's diseases [27].

In their study, Ozcimen et al. reported significantly lower mean RFNL thickness in COPD cases, although no comparison was made for the different quadrants [8]. Bayhan et al. studied RFNL thickness in cases with obstructive

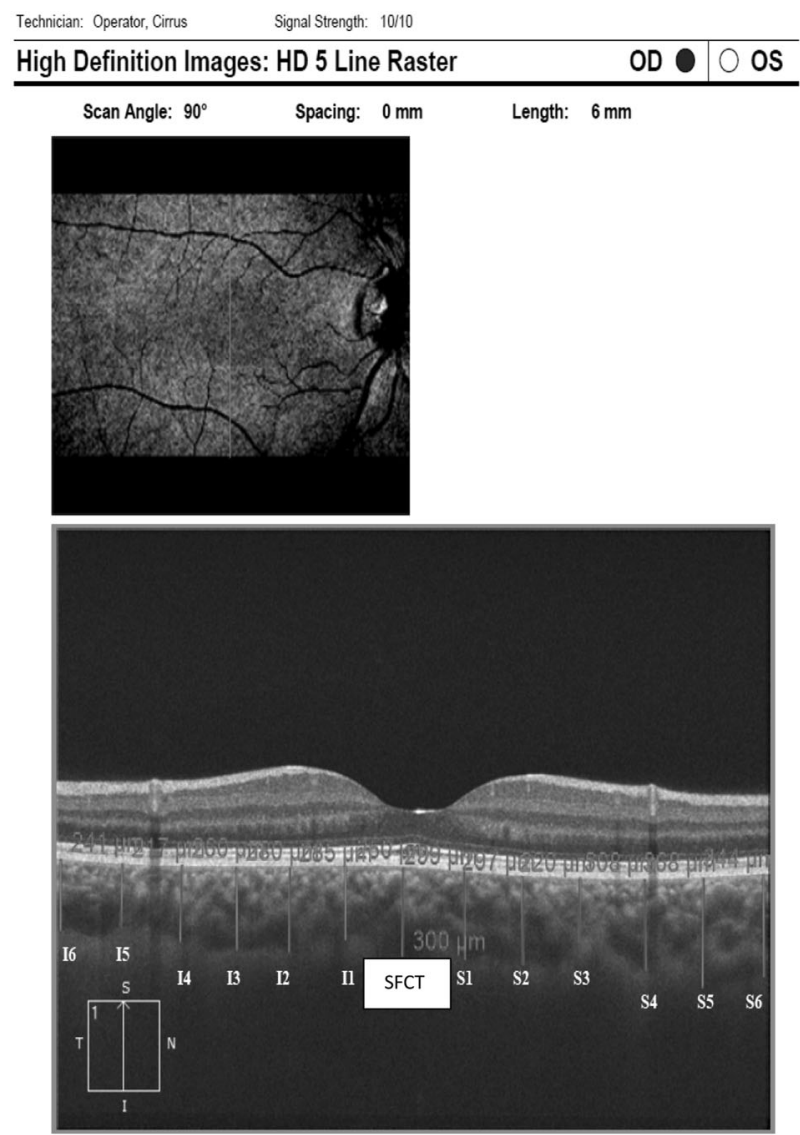

Fig. 3 An example of EDI-OCT examination of Subfoveal choroidal thickness measurement of a patient

sleep apnea syndrome and determined significant thinning in the nasal quadrant [6].

Average RFNL measurements in our study were found to be lower in severe COPD patients when compared with control group but this difference was not statistically significant. This measurement was also determined to be lowered from the initial values measured in mild-modere or severe COPD groups respectively when measured after 3 months from the initial visit, but this difference was not statistically significant either. FVC and FEV1 values have a positive correlation with ARNFL in even mild cases of COPD. In severe cases of COPD, number of smoked cigarettes (packets) per year and attacks per year are found to have a negative correlation with ARNFL values-which indicate the possible role of hypoxia in thinning of ARNFL measurements of these cases.

Inferior quadrant RNFL values were significantly lower in severe COPD patients in comparison with control group patients, both in the initial and 3rd month examinations. Temporal quadrant RNFL values were also found to be reduced significantly in severe COPD group than mild-moderate COPD group in the 3rd month examination of the cases. This may be a possible result of chronic 
Fig. 4 Subfoveal choroidal thickness (SFCT) measurements of a patient in Group 1 (GOLD A-B) in first and 3rd month examination
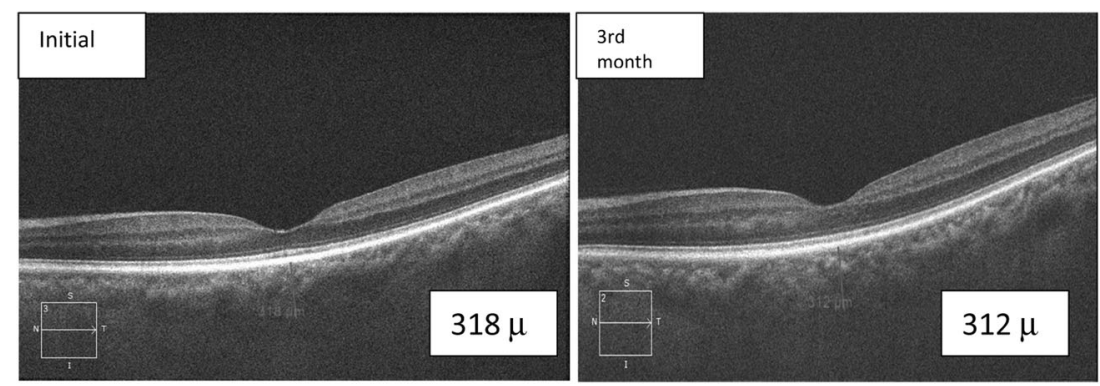

Fig. 5 Subfoveal choroidal thickness (SFCT) measurements of a patient in Group 2 (GOLD $\mathrm{C}-\mathrm{D}$ ) in first and 3rd month examination
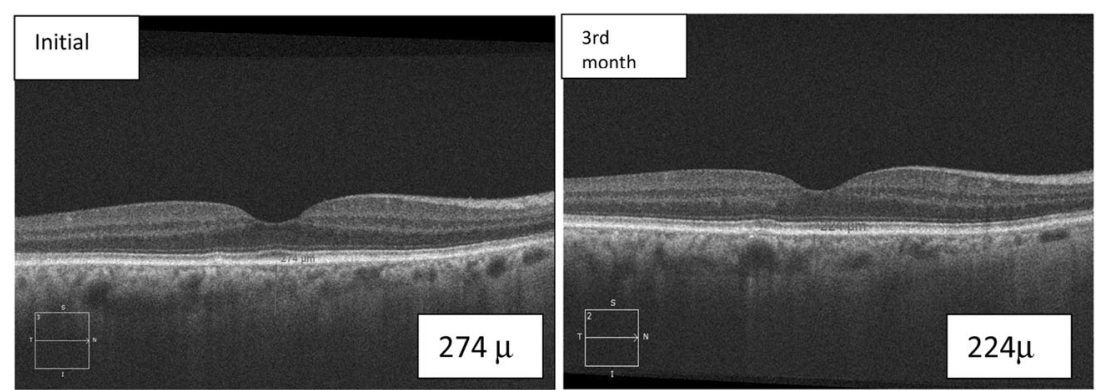

hypoxia and systemic inflammation triggered by the disease, cytokin release, and disturbed oxidant/antioxidant balance $[29,30]$.

The role of hypoxia has been already demonstrated in many studies in axonal loss and death of ganglion cells $[27,28,31]$. Inner layers of retina, especially the ganglion cells are vulnerable to any kind of ischemic insult, such as hypoxic conditions [27, 32]. Abnormalities in ocular blood flow (e.g., increased resistance), systemic inflammation, increase in endothelin-like systemic vasoconstrictor levels, and nocturnal hypoxia resulting in neuronal damage over the axons are proposed mechanisms of the progressive RNFL and GCC loss in COPD patients [13].

Average GCC analysis of severe COPD patients in our study were found to be significantly lower when compared with age and sex-matched control group-both in the initial and 3rd month examination. The deterioration in GCC analysis during the 3-month follow-up of these severe cases was significantly more prominent than mild COPD and control group cases. This demonstrates that a subclinical neurodegenerative effect of the chronic hypoxia exists on retinal neurons and axons. Since the severe COPD group consists of patients with a greater number of hypoxic attacks in history and a lower oxygen saturation on daily basis, progressive loss of RNFL and GCC are inevitable results of the condition. These findings were supported by the correlation analysis, indicating that the COPD duration and number of attacks per year are found to have a significant negative correlation on Average GCC analysis results in severe COPD cases, in both initial and 3rd month controls.

One of the major restrictions of our study was limited number of relevant subjects. Patients with COPD commonly have coexisting systemic conditions, such as diabetes mellitus, coronary heart disease, and congestive heart failure. These systemic disorders might interfere with OCT examination results, so these subjects had to be excluded from the study. Severe COPD subjects that are dependent on invasive or noninvasive mechanical ventilation also had to be excluded from the study, so number of the study participants were limited.

Controlled design of the study with age, and sexmatched control group and the sequential analysis of retina and choroid of COPD patients with different severities, both in the initial and 3rd month examinations are valuable aspects of our study. There is no similar study in the literature that inspected the serial changes in OCT parameters of COPD patient, during any period of follow up.

In the light of our study, it is evident that COPD patients should be evaluated in more details in terms of accompanying ocular pathologies that appear as a result of systemic hypoxemia. New randomized- and larger-sized controlled trials on the correlation of hypoxia and OCT measurements; and the effects of the chronic hypoxic status of COPD patients on ocular tissues should be carried on in the future.

\section{Summary}

\section{What was known before}

- The eye seems to be one of the affected tissues of the COPD with hypoxia. 


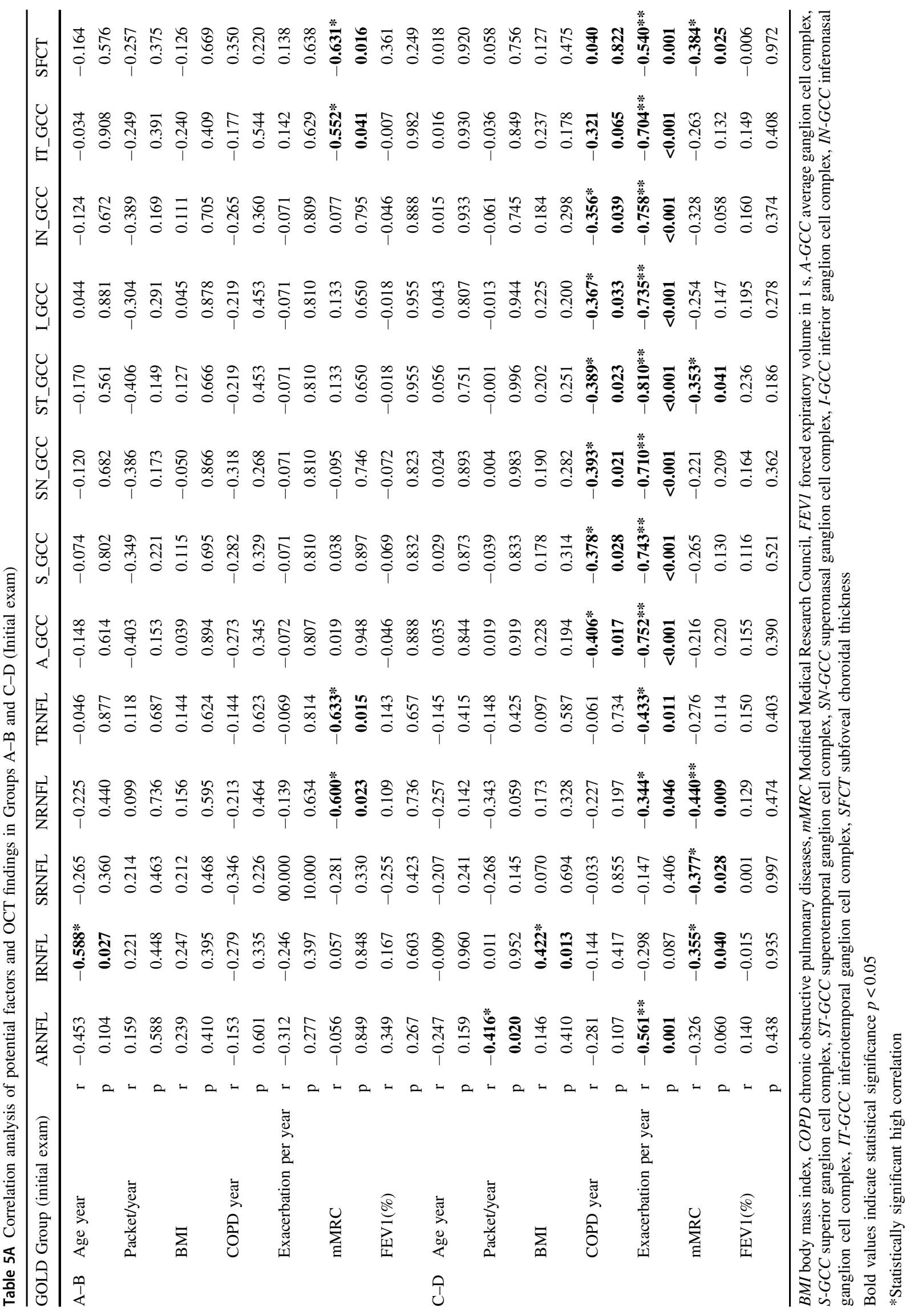




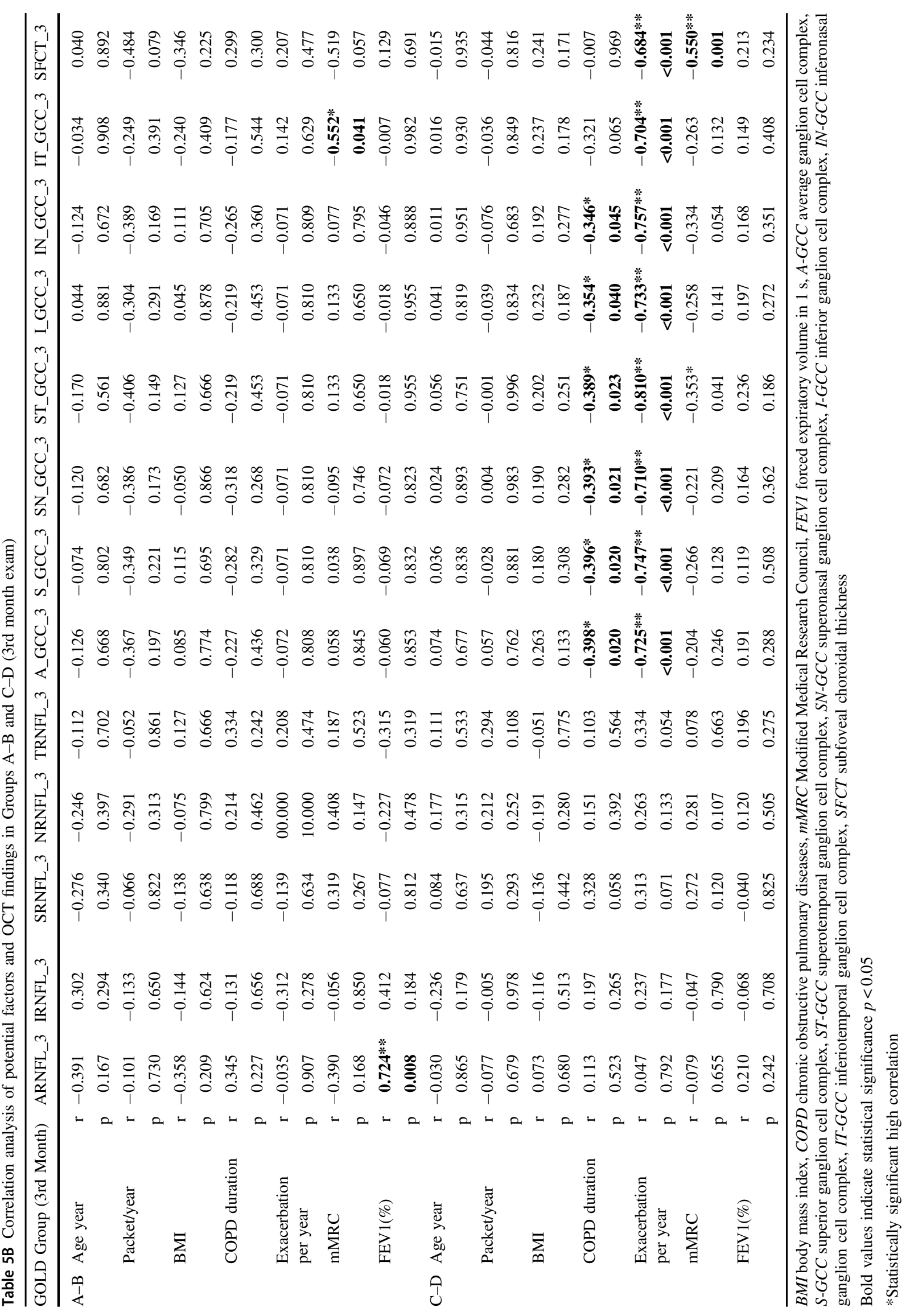




\section{What this study adds}

- Hypoxia is thought to be the underlying mechanism in COPD which may influence retinal and choroidal OCT parameters.

- Decrease in blood flow of optic nerve head, increased vascular resistance and reduced blood flow in choroid may affect the visual ability in these patients, which should be kept in mind during their follow-up.

\section{Compliance with ethical standards}

Conflict of interest The authors declare that they have no conflict of interest.

Ethical standards The study was conducted in accordance with the ethical standards of the institutional and/or national research committee and with the 1964 Helsinki declaration and its later amendments or comparable ethical standards.

Informed consent Informed consent was obtained from all individual participants included in the study

Publisher's note Springer Nature remains neutral with regard to jurisdictional claims in published maps and institutional affiliations.

\section{References}

1. Asia Pacific COPD Roundtable Group. Global Initiative for chronic obstructive lung disease strategy for the diagnosis, management and prevention of chronic obstructive pulmonary disease: an Asia-Pacific perspective. Respirology. 2005;10:9-17.

2. Gan WQ, Man SF, Senthilselvan A, Sin DD. Association between chronic obstructive pulmonary disease and systemic inflammation: a systematic review and a meta-analysis. Thorax. 2004;59:574-80.

3. Wouters EF, Creutzberg EC, Schols AM. Systemic effects in COPD. Chest. 2002;121:127-30.

4. Vernooy JH, Kucukaycan M, Jacobs JA, Chavannes NH, Buurman WA, Dentener MA, et al. Local and systemic inflammation in patients with chronic obstructive pulmonary disease: soluble tumor necrosis factor receptors are increased in sputum. Am J Respir Crit Care Med. 2002;166:1218-24.

5. Rabe KF, Hurd S, Anzueto A, Barnes PJ, Buist SA, Calverley P, et al. Global initiative for chronic obstructive lung disease. Global strategy for the diagnosis, management, and prevention of chronic obstructive pulmonary disease: GOLD executive summary. Am J Respir Crit Care Med. 2007;176:532-55.

6. Bayhan HA, Aslan Bayhan S, İntepe YS, Muhafiz E, Gürdal C. Evaluation of the macular choroidal thickness using spectral optical coherence tomography in patients with obstructive sleep apnoea syndrome. Clin Exp Ophthalmol. 2015;43:139-44.

7. Xin C, Wang J, Zhang W, Wang L, Peng X. Retinal and choroidal thickness evaluation by SD-OCT in adults with obstructive sleep apnea-hypopnea syndrome (OSAS). Eye. 2014;28:415-21.

8. Ozcimen M, Sakarya Y, Kurtipek E, Bekci TT, Goktas S, Sakarya R, et al. Peripapillary choroidal thickness in patients with chronic obstructive pulmonary disease. Cutan Ocul Toxicol. 2016;35:26-30.
9. Jaffe G-J, Caprioli J. Optical coherence tomography to detect and manage retinal disease and glaucoma. Am J Ophthalmol. 2004;137:156-69.

10. Adhi M, Duker JS. Optical coherence tomography-current and future applications. Curr Opin Ophthalmol. 2013;24:213-21.

11. Koh VT, Tham YC, Cheung CY, Wong WL, Baskaran M, Saw $\mathrm{SM}$, et al. Determinants of ganglion cell-inner plexiform layer thickness measured by high-definition optical coherence tomography. Investig Ophthalmol Vis Sci. 2012;53:5853-9.

12. Ugurlu E, Pekel G, Altinisik G, Bozkurt K, Can I, Evyapan F.New aspect for systemic effects of COPD: eye findings.Clin Respir J. 2018;12:247-52.

13. Gok M, Ozer MA, Ozen S, Botan Yildirim B. The evaluation of retinal and choroidal structural changes by optical coherence tomography in patients with chronic obstructive pulmonary disease. Curr Eye Res. 2018;43:116-21.

14. -Pauwels RA, Buist AS, Calverley PM, Jenkins CR, Hurd SS, GOLD Scientific Committee. Global strategy for the diagnosis, management, and prevention of chronic obstructive pulmonary disease NHLBI/WHO Global Initiative for Chronic Obstructive Lung Disease (GOLD) Workshop summary. Am J Respir Crit Care Med. 2001;163:1256-76.

15. Kang HM, Kwon HJ, Yi JH, Lee CS, Lee SC. Subfoveal choroidal thickness as a potential predictor of visual out- come and treatment response after intravitreal ranibizumab injections for typical exudative age-related macular degeneration. Am J Ophthalmol. 2014;157:1013-21.

16. Rayess N, Rahimy E, Ying GS, Bagheri N, Ho AC, Regillo CD, et al. Baseline choroidal thickness as a predictor for response to anti-vascular endothelial growth factor therapy in diabetic macular edema. Am J Ophthalmol. 2015;159:85-91.

17. Manjunath V, Taha M, Fujimoto JG, Duker JS. Choroidal thickness in normal eyes measured using cirrus HD optical coherence tomography. Am J Ophthalmol. 2010;150:325-9.

18. Xu J, Xu L, Du KF, Shao L, Chen CX, Zhou JQ, et al. Subfoveal choroidal thickness in diabetes and diabetic retinopathy. Ophthalmology. 2013;120:2023-8.

19. Wong IY, Wong RL, Zhao P, Lai WW. Choroidal thickness in relation to hypercholesterolemia on enhanced depth imaging optical coherence tomography. Retina. 2013;33:423-8.

20. Pekel G, Akin F, Erturk MS, Acer S, Yagci R, Hiraali MC, et al. Chorio-retinal thickness measurements in patients with acromegaly. Eye. 2014;28:1350-4.

21. Ingegnoli F, Gualtierotti R, Pierro L, Del Turco C, Miserocchi E, Schioppo T, et al. Choroidal impairment and macular thinning in patients with systemic sclerosis: the acute study. Microvasc Res. 2014;97:31-6.

22. Rishi P, Rishi E, Mathur G, Raval V. Ocular perfusion pressure and choroidal thickness in eyes with polypoidal choroidal vasculopathy, wet-age-related macular degeneration, and normal. Eye. 2013;27:1038-43.

23. Ahmad M, Kaszubski PA, Cobbs L, Reynolds H, Smith RT Choroidal thickness in patients with coronary artery disease. PLoS ONE. 2017;12:e175691.

24. Ahn SJ, Woo SJ, Park KH. Retinal and choroidal changes with severe hypertension and their association with visual out- come. Investig Ophthalmol Vis Sci. 2014;55:7775-85.

25. Altinkaynak H, Kara N, Sayin N, Gunes H, Avsar S, Yazici AT. Subfoveal choroidal thickness in patients with chronic heart failure analyzed by spectral-domain optical coherence tomography. Curr Eye Res. 2014;39:1123-8.

26. Dinh-Xuan AT, Higenbottam TW, Clelland CA, Stiebellehner L, Petkov V, Schenk P, et al. Impairment of endothelium-dependent pulmonary-artery relaxation in chronic obstructive lung disease. $\mathrm{N}$ Engl J Med. 1991;324:1539-47. 
27. Lamirel C, Newman NJ, Biousse V. Optical coherence tomography (OCT) in optic neuritis and multiple sclerosis. Rev Neurol. 2010;166:978-86.

28. Sakai RE, Feller DJ, Galetta KM, Galetta SL, Balcer LJ. Vision in multiple sclerosis (MS): the story, structure-function correlations, and models for neuroprotection. J Neuroophthalmol. 2011;31:362-73.

29. Evans RA, Morgan MD. The systemic nature of chronic lung disease. Clin Chest Med. 2014;35:283-93.
30. Domej W, Oettl K, Renner W. Oxidative stress and free radicals in COPD implications and relevance for treatment. Int $\mathrm{J}$ Chron Obstruct Pulmon Dis. 2014;9:1207-24.

31. Palombi K, Renard E, Levy P, Chiquet C, Deschaux Ch, Romanet $\mathrm{JP}$, et al. Non-arteritic anterior ischaemic optic neuropathy is nearly systematically associated with obstructive sleep apnoea. $\mathrm{Br}$ J Ophthalmol. 2006;90:879-82.

32. Kergoat H, Herard ME, Lemay M. RGC sensitivity to mild systemic hypoxia. Investig Ophthalmol Vis Sci. 2006;47:5423-7. 